\title{
Cyclone Idai as a Trigger for Pellagra Outbreak in Nhamatanda, Mozambique: A Case-Control Study
}

\author{
Vánio André Mugabe, ${ }^{1,2 \star}$ Arlete Mahumane,${ }^{3}$ Cynthia Semá Baltazar, ${ }^{4}$ Érika Valeska Rossetto, ${ }^{5}$ Crescêncio Sequeira Nhabomba, ${ }^{3}$ \\ Neusa Fataha, ${ }^{4}$ Unicia Nyamula, ${ }^{3}$ Angélica Sotomane, ${ }^{3}$ Wilson Irugula, ${ }^{6}$ Benigno Canze, ${ }^{3}$ Osvaldo Frederico Inlamea, ${ }^{4}$ \\ Uriel Kitron, ${ }^{7,8}$ Guilherme Sousa Ribeiro, ${ }^{1,7,9 *}$ and Eduardo Samo Gudo ${ }^{4}$ \\ ${ }^{1}$ Instituto de Saúde Coletiva, Universidade Federal da Bahia, Salvador, Brazil; ${ }^{2}$ Universidade Licungo, Quelimane, Mozambique; ${ }^{3}$ Centro de \\ Investigação Operacional da Beira, Instituto Nacional de Saúde, Beira, Mozambique; ${ }^{4}$ Departamento de Vigilância, Instituto Nacional de Saúde, \\ Maputo, Mozambique; ${ }^{5}$ MassGenics, Assigned to Mozambique Centers for Disease Control Prevention, Maputo, Mozambique; ${ }^{6}$ Direcção \\ Provincial de Saúde de Sofala, Beira, Mozambique; ${ }^{7}$ Fundação Oswaldo Cruz, Salvador, Brazil; ${ }^{8}$ Emory University, Atlanta, Georgia; ${ }^{9}$ Faculdade de \\ Medicina, Universidade Federal da Bahia, Salvador, Brazil
}

\begin{abstract}
In mid-June 2019, 3 months after cyclone Idai landfall in Mozambique, health authorities of Nhamatanda district reported an outbreak of Pellagra. Applying a mixed-method protocol, we carried out an investigation to characterize cases of pellagra, identify the associated factors for the outbreak using a case-control study, and explore the perceived impact on food security (availability, access, and usage) before and after Idai. We collected data from $121 \mathrm{cases}$ and 121 controls and conducted in-depth interviews with 69 heads of households. The cases were more likely to be female $(P<0.01)$ and less educated $(P<0.01)$ than controls. Insufficient consumption of chicken and peanut before cyclone Idai arrival were statistically associated with pellagra $(P<0.05)$. From interviewed households' heads, $51 \%$ were experiencing food shortages even before the cyclone hit. Cyclone ldai served as a trigger to reduce niacin consumption below the threshold that protected Nhamatanda population from pellagra and caused $a \approx 2,300$ case $(707.9 / 100,000$ inhabitants) outbreak.
\end{abstract}

On March 14, 2019, Idai, a very powerful cyclone (level 4 in the 1-5 Saffir-Simpson scale ${ }^{1}$ ), reached mainly the districts of Beira, Búzi, Dondo, and Nhamatanda, in Sofala Province, Mozambique, and was followed by heavy rainfalls and flooding. It destroyed agricultural assets, livestock, and fisheries and threatened the food security of hundreds of thousands of people across the four districts. In mid-June 2019, health authorities of Nhamatanda district reported a pellagra outbreak ( $\approx 100$ cases) among people living in/or around resettlement centers hosting displaced people. Nhamatanda $\left(\approx 322,511\right.$ inhabitants; $\left.2017^{2}\right)$ is one of the poorest districts in Sofala Province; most inhabitants are small farmers practicing subsistence agriculture and petty traders. The district is self-sufficient in the production of corn, the region's staple food.

Pellagra is a disease caused by a deficiency of niacin (vitamin B3) and tryptophan in the body, due to either malnutrition or absorption problems. ${ }^{3-5}$ The characteristic manifestations are dermatitis, diarrhea, and dementia. If untreated, it can lead to death. 6,7 The diagnostic is based on dermatological signs and can be confirmed by low levels of niacin metabolites in urine. Worldwide, pellagra was a public health problem until mid-twentieth century. Currently, it is an uncommon disease, due to the consumption of enriched diets with vitamins and/or consumption of nixtamalized maize (a cooking process for preparation of corn that increases the availability of niacin) where corn is a staple food. ${ }^{8-10}$

Between June 29 and July 19, we investigated the pellagra outbreak in Nhamatanda district, applying a mixed-method protocol to characterize cases of pellagra, identify factors associated with case occurrence, and explore the perceived impact on food security (availability, access, and usage) before and after Idai arrival using an in-depth interview. The

\footnotetext{
*Address correspondence to Vánio André Mugabe, Universidade Licungo, Estrada Regional, n 642, PC 106, Quelimane, Mozambique, E-mail: vandremu@gmail.com or Guilherme Sousa Ribeiro, Oswaldo Cruz Foundation, Brazilian Ministry of Health, Rua Waldemar Falcão, 121, Candeal - CEP: 40296-710, Salvador, Brazil, E-mail: guilherme.ribeiro@ fiocruz.br.
}

Ethical Research Committee of the National Institute of Health of Mozambique granted ethical approval, and oral informed consent was obtained.

An unmatched case-control study aimed to include 138 cases and 138 controls to provide $80 \%$ power to detect an odds ratio $(\mathrm{OR}) \geq 2$ using a $5 \%$ significance level. Cases were defined clinically by the presence of bilateral and symmetrical dermatitis in body regions exposed to sunlight (face, lips, hands, arms, and legs), or typical Casal's necklace. Cases were identified in the district medical records or through active case searching in hamlets, followed by clinical diagnosis from experts. Individuals without dermatological features and not using a vitamin supplement 30 days before enrollment, who lived in proximity to each case, served as controls; they were chosen based on proximity to the age-group ( $<5,5-14$, and $>14$ years) of the cases.

Participants were visited once at their homes. Through faceto-face interviews, we recorded sociodemographic and epidemiological data and potential risk factors based on dietary patters from January through mid-March (before Idai arrival) and from mid-March to July (after Idai landfall). Parents and/or legal guardians provided answers for their children. Interviews were translated into Sena (the local language) as needed. Characteristics of cases and controls, including food consumption patterns before and after Idai arrival, were compared using a chi-square test or Fisher's exact test. Each variable with $P<0.25$ was adjusted for gender in the multivariable logistic regression, considering a two-tailed $P$-value $<0.05$.

Because of difficulties in simultaneously coordinating the inclusion of participants in the study, while providing assistance to the population, we terminated participants' enrollment earlier, with a total of 121 cases and 121 controls from nine hamlets. Among cases, 110 (90.9\%) were women and six $(5 \%)$ were children $<5$ years. Of 99 women aged $\geq 15$ years, eight $(8.1 \%)$ were pregnant and 48 (48.5\%) were breastfeeding. Dermatitis (rough skin) appeared on arms (92.0\%), face (41.6\%), neck (40.7\%), lips (23.0\%), chest (5.3\%), and legs (2.7\%) (Figure 1). And $6.6 \%$ of cases had stomatitis and $3.3 \%$ 

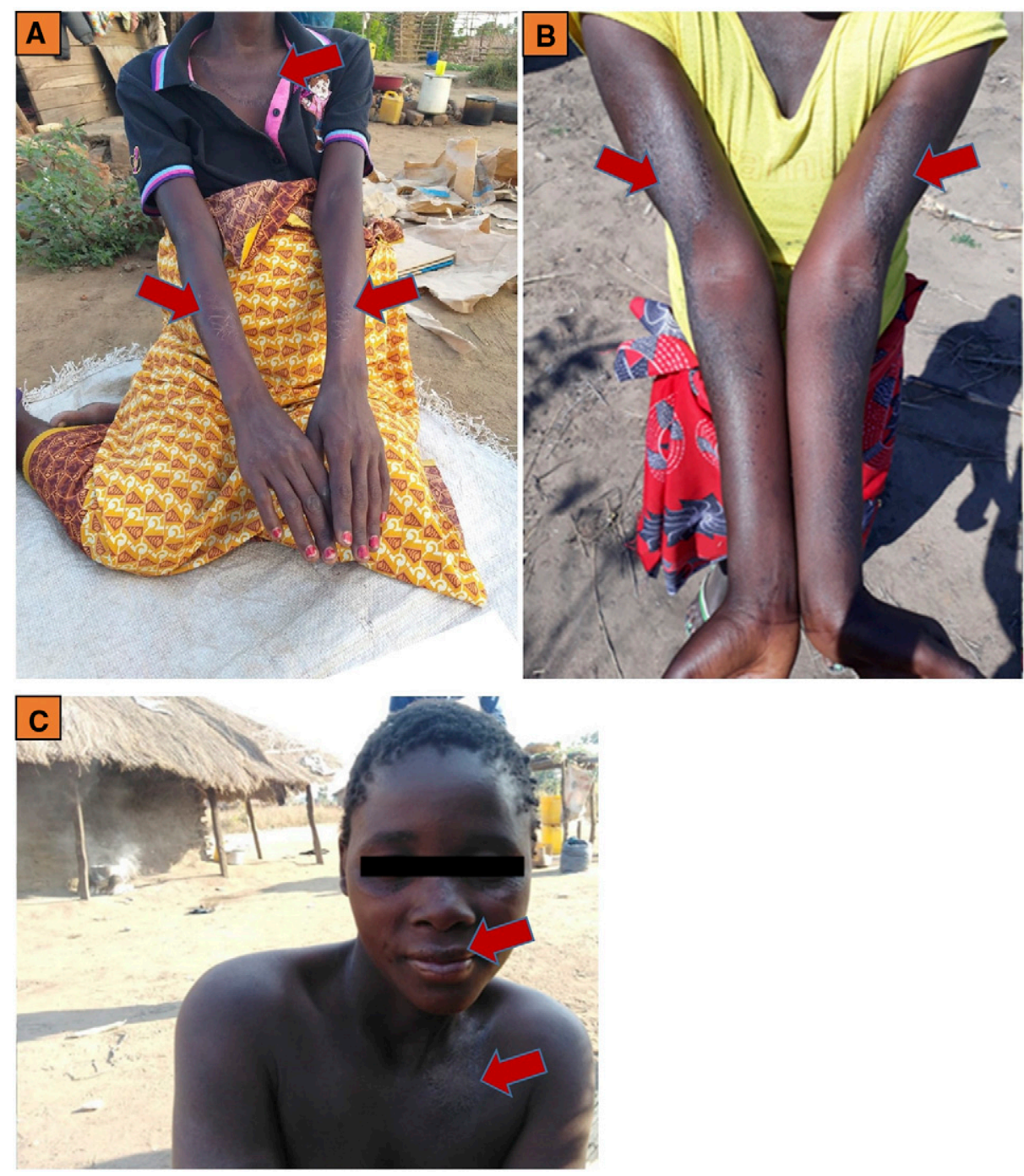

FIGURE 1. Pictures of patients with dermatological signs characteristic of pellagra at the time of case detection during an outbreak in Mozambique. Arrows indicate typical dermatological features of pellagra cases identidied during the outbreak in Mozambique. Skin lesions are observed in (A and B) arms, (A, B, and C) chest (with typical Casal's neck appearance), and (C) face. This figure appears in color at www.ajtmh.org.

diarrhea, and none was hospitalized. Cases were more likely to be female $(P<0.01)$ and less educated $(P<0.01)$ than controls (Table 1). Insufficient consumption of chicken (genderadjusted OR $=2.04 ; 95 \% \mathrm{Cl}: 1.16-3.58$ ) and peanut (genderadjusted $\mathrm{OR}=1.8 ; 95 \% \mathrm{Cl}: 1.02-3.17$ ) before cyclone ldai arrival was associated with pellagra (Table 2).

We performed in-depth interviews with 69 heads of households from six hamlets with high frequency of pellagra. To assess food security, we used questions adapted from "the U.S. Adult Food Security Survey Module."11 The key questions asked were "Is the food you buy enough to feed the family for a month?" "Are you worried that the food would run out before you can get money to buy more?" and "Have you or other adults in the family reduced your meal or skipped a meal because you do not have enough food? Households were classified as short on food, if respondents answered that they do not get enough food, they usually or sometimes worry about running out of food, or if they skip meals or reduce the amount of food during meals.

The median family size was six (interquartile range: 5-8) members. Over half (51\%) of the households were experiencing food shortages, usually consuming only 1-2 meals a day, with very limited diversity and no snacks in between. The remaining households still felt that food quantity and quality were lacking. Poverty, characterized by low education (only $8.7 \%$ of households have at least one member with high school education), low purchasing power (all were resource-poor farmers or fishers), and few job opportunities (none had a formal job), makes survival challenging. Respondents revealed that they do not produce enough for their own year-round consumption and some sell the crop to cover other needs. Food stocks are generally exhausted in November, and people often go hungry from January until the harvest in April. During the famine period, some people develop "bacalhau" (local name for pellagra): rough and dry skin that looks like dried codfish (also bacalhau in Portuguese).

When cyclone Idai arrived and floods washed everything away, many households were already starving because of low harvest in previous years. Some people remained without food for up to 4 days until the arrival of food aid (essentially rice, corn flour, sugar, peas, cooking oil, pasta, sardines, and soy flour) distributed by humanitarian agencies. Because food 
TABLE 1

Characteristics of pellagra cases and controls from the general population, Nhamatanda, 2019

\begin{tabular}{|c|c|c|c|}
\hline \multirow[b]{2}{*}{ Explanatory variable } & Cases with pellagra $(N=121)$ & Controls from general population $(N=121)$ & \multirow[b]{2}{*}{$P$-value } \\
\hline & \multicolumn{2}{|c|}{ Number (\%) } & \\
\hline \multicolumn{4}{|c|}{ Demographic characteristics } \\
\hline \multicolumn{4}{|c|}{ Gender } \\
\hline Male & $11(9.1)$ & $54(44.6)$ & \multirow[t]{2}{*}{$<0.01$} \\
\hline Female & $110(90.9)$ & $67(55.4)$ & \\
\hline \multicolumn{4}{|l|}{ Age (years) } \\
\hline $0-4$ & $6(5.0)$ & $5(4.1)$ & \multirow[t]{3}{*}{$1.00^{\star}$} \\
\hline $5-14$ & $10(8.3)$ & $10(8.3)$ & \\
\hline$>14$ & $105(87.6)$ & $106(87.6)$ & \\
\hline \multicolumn{4}{|c|}{ No. of individuals per family. } \\
\hline $1-5$ & $44(36.4)$ & $41(33.8)$ & \multirow[t]{3}{*}{$0.87^{\star}$} \\
\hline $6-10$ & $65(53.7)$ & $66(54.6)$ & \\
\hline$>10$ & $12(9.9)$ & $14(11.6)$ & \\
\hline \multicolumn{4}{|c|}{ Monthly family income in US\$ } \\
\hline$\leq 15.99$ & $90(74.4)$ & $86(71.1)$ & \multirow[t]{3}{*}{0.84} \\
\hline$>16.00$ and $\leq 47.95$ & $20(16.5)$ & $22(18.2)$ & \\
\hline$>47.95$ & $11(9.1)$ & $13(10.7)$ & \\
\hline \multicolumn{4}{|l|}{ Education level } \\
\hline Illiterates & $48(39.7)$ & $26(21.5)$ & \multirow[t]{3}{*}{$<0.01$} \\
\hline Primary & $62(51.2)$ & $74(61.2)$ & \\
\hline Secondary & $11(9.1)$ & $21(17.3)$ & \\
\hline \multicolumn{4}{|l|}{ Employment } \\
\hline No & $120(99.2)$ & $116(95.9)$ & \multirow[t]{2}{*}{0.10} \\
\hline Yes & $1(0.8)$ & $5(4.1)$ & \\
\hline \multicolumn{4}{|l|}{ Self-reported health status } \\
\hline HIV status & & & \multirow{4}{*}{0.29} \\
\hline Negative & $53(43.8)$ & $54(44.6)$ & \\
\hline Positive & 15 (12.4) & $8(6.6)$ & \\
\hline Unaware & $53(43.8)$ & $59(48.8)$ & \\
\hline \multicolumn{4}{|l|}{ TB status } \\
\hline Negative & $120(99.2)$ & $119(98.3)$ & \multirow[t]{2}{*}{$1.00^{\star}$} \\
\hline Positive & $1(0.8)$ & $2(1.7)$ & \\
\hline \multicolumn{4}{|l|}{ Cyclone-related features } \\
\hline Got sick after cyclone & & & \\
\hline No & $50(41.3)$ & $61(50.4)$ & \multirow[t]{2}{*}{0.16} \\
\hline Yes & $71(58.7)$ & $60(49.6)$ & \\
\hline \multicolumn{4}{|l|}{ Resettled after cyclone } \\
\hline No & $114(94.2)$ & $110(90.9)$ & \multirow[t]{2}{*}{0.33} \\
\hline Yes & $7(5.8)$ & $11(9.1)$ & \\
\hline Food aid after cyclone & & & \\
\hline No & $2(1.7)$ & $3(2.5)$ & $1.00^{*}$ \\
\hline Yes & $119(98.3)$ & $118(97.5)$ & \\
\hline Food offer & & & \\
\hline Increased & $2(1.7)$ & $8(6.6)$ & 0.15 \\
\hline Unchanged & $15(12.3)$ & $15(12.4)$ & \\
\hline Decreased & $104(86.0)$ & 98 (81.0) & \\
\hline
\end{tabular}

supply decreased over time, hunger worsened, and some households resorted to wetted and spoiled corn in the fields to supply their food needs. For long-term recovery, humanitarian agencies supported communities through restocking domestic poultry and distributing seeds (maize, sesame, peanuts, beans, and vegetables seeds). However, the rains that fell earlier this year have once again destroyed crops in the fields.

The outbreak of pellagra in Nhamatanda is the second recorded in Mozambique, after the first one affecting civil war refugees returning from Malawi to Tete Province in $1995 .^{12}$ During the Idai emergency, the first cases of pellagra were detected by the outpatient clinic ran by Medicos Del Mundo, an association that aims to provide the right to health, especially for vulnerable populations, such as victims of natural disasters. Then, they initiated extensive active pellagra surveillance across Nhamatanda district and trained health technicians from other districts, also affected by cyclone Idai, to detect potential cases. Between July 2019 and March
2020, the health system registered 3,590 (302.9/100,000 inhabitants) people with pellagra. Of these, 2,283 (63\%) were recorded in Nhamatanda $(707.9 / 100,000$ inhabitants) (Figure 2).

For short-term treatment, doctors used vitamin B complex pills and offered four packs ( $50 \mathrm{~g}$ each) of roasted peanuts and a packet of niacin-enriched biscuits per patient; health status improved a week after starting the treatment, confirming that the disease was due to malnutrition and vitamin deficiency. Poverty, natural disasters, climatic catastrophe, wars, migration, and refugee settings are the main fuel for pellagra outbreaks ${ }^{3,13,14}$ as they limit the availability and access to food in quantity and quality necessary to feed affected people. Although food aid relieved the impact of hunger, it failed to provide enough essential nutrients. In Malawi (1989/90) and Angola (2000), vitamin B complex and groundnuts were included in the food basket as a preventive measure for pellagra during outbreaks. $3,13,14$ 
TABLE 2

Odds ratios for associations between the pattern of food consumption before and after cyclone Idai arrival and pellagra in Nhamatanda, 2019

\begin{tabular}{|c|c|c|c|c|}
\hline \multirow[b]{2}{*}{ Explanatory variable } & Cases $(n=121)$ & Controls $(n=121)$ & \multirow[b]{2}{*}{ Crude OR (95\% Cl) } & \multirow[b]{2}{*}{ Gender-adjusted OR (95\% Cl) } \\
\hline & \multicolumn{2}{|c|}{ Number (\%) } & & \\
\hline \multicolumn{5}{|l|}{ Before cyclone Idai landfall } \\
\hline Twice a week or more* & $4(3.3)$ & $3(2.5)$ & $1.34(0.22 ; 9.37) \dagger$ & - \\
\hline \multicolumn{5}{|l|}{ Corn consumption } \\
\hline $\begin{array}{l}\text { Four times a week or moreł } \\
\text { Meat consumption }\end{array}$ & $106(87.6)$ & $106(87.6)$ & $1.00(0.47 ; 2.15)$ & - \\
\hline Once a month or never§ & $99(81.8)$ & $85(70.3)$ & $1.91(1.04 ; 3.49)$ & $1.91(0.99 ; 3.68)$ \\
\hline \multicolumn{5}{|l|}{ Milk consumption } \\
\hline $\begin{array}{l}\text { Once a month or never§ } \\
\text { Eg consumption }\end{array}$ & $114(94.2)$ & $111(91.7)$ & $1.47(0.54 ; 3.99)$ & - \\
\hline Once a month or never§ & $86(71.1)$ & $69(57.0)$ & $1.85(1.09 ; 3.16)$ & $1.74(0.98 ; 3.11)$ \\
\hline \multicolumn{5}{|l|}{ Chicken consumption } \\
\hline \multirow{2}{*}{\multicolumn{5}{|c|}{ Peanut consumption }} \\
\hline & & & & \\
\hline Once a month or never§ & $58(47.9)$ & $42(34.7)$ & $1.73(1.03 ; 2.90)$ & $1.8(1.02 ; 3.17)$ \\
\hline \multicolumn{5}{|l|}{ After cyclone Idai landfall } \\
\hline \multicolumn{5}{|l|}{ Alcohol consumption } \\
\hline Twice a week or more ${ }^{\star}$ & $4(3.3)$ & $3(2.5)$ & $1.34(0.22 ; 9.37) \dagger$ & - \\
\hline \multicolumn{5}{|l|}{ Corn consumption } \\
\hline Four times a week or moreł & $57(47.1)$ & $58(47.9)$ & $0.97(0.58 ; 1.60)$ & - \\
\hline \multicolumn{5}{|l|}{ Meat consumption } \\
\hline Once a month or never§ & $115(95.0)$ & $108(89.3)$ & $2.31(0.85 ; 6.29)$ & $2.15(0.73 ; 6.33)$ \\
\hline \multicolumn{5}{|l|}{ Milk consumption } \\
\hline Once a month or never§ & $118(97.5)$ & $114(94.2)$ & $2.42(0.53 ; 14.77) \dagger$ & - \\
\hline \multicolumn{5}{|l|}{ Egg consumption } \\
\hline Once a month or never§ & $109(90.1)$ & $105(86.8)$ & $1.38(0.63 ; 3.07)$ & - \\
\hline \multicolumn{5}{|l|}{ Chicken consumption } \\
\hline Once a month or never§ & $107(88.4)$ & $101(83.5)$ & $1.51(0.73 ; 3.16)$ & - \\
\hline \multicolumn{5}{|l|}{ Peanut consumption } \\
\hline Once a month or never§ & $86(71.1)$ & $75(62.0)$ & $1.51(0.88 ; 2.58)$ & $1.49(0.83 ; 2.68)$ \\
\hline
\end{tabular}

Our case-control study points to gender (female) and education level (low) as factors associated with pellagra, in agreement with findings from other countries. ${ }^{15}$ The increased odds in women may be explained partly by cultural (women depriving themselves of food to feed their children or husbands) and physiological (hormone peaks and higher bodily requirements during pregnancies and breastfeeding) causes. ${ }^{15-19}$

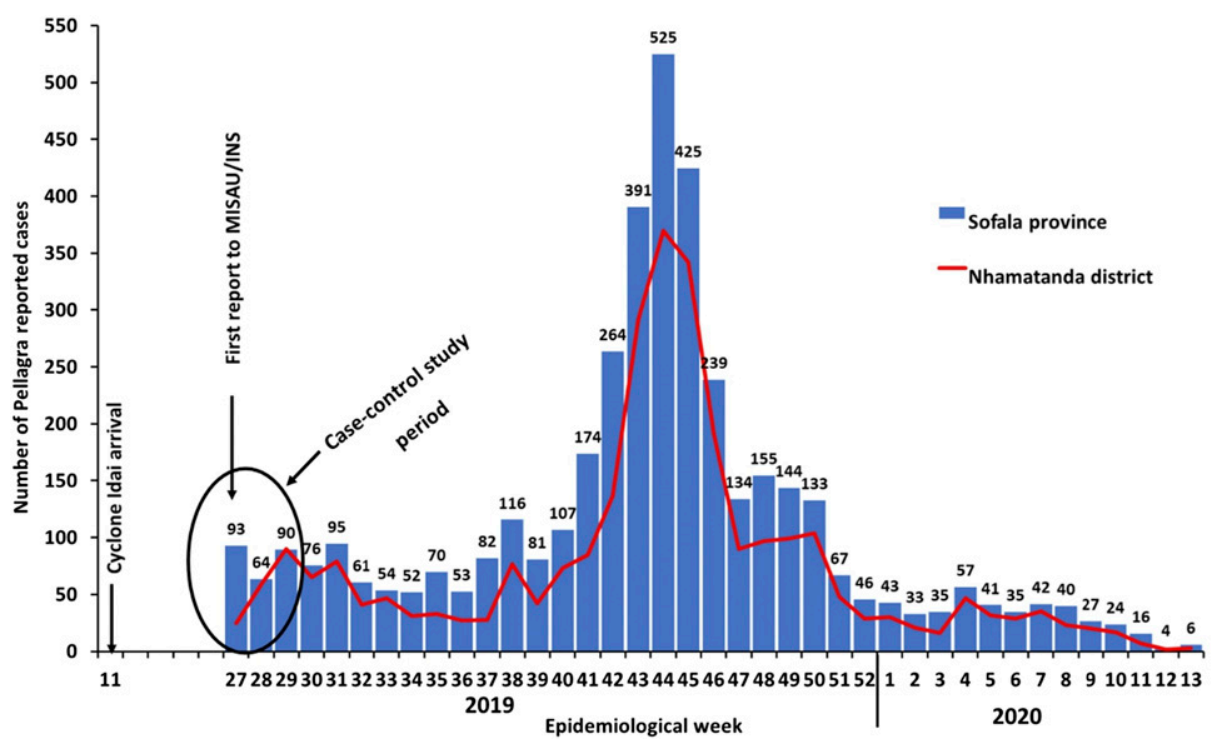

FIGURE 2. Temporal distribution of pellagra cases reported through passive surveillance in Sofala Province and through active surveillance in the district of Nhamatanda, Mozambique, between July 2019 and March 2020. Source of data: mAlert platform, a Web-based platform hosted by the Ministry of Health of Mozambique/National Institute of Health. This figure appears in color at www.ajtmh.org. 
The study had limitations. Logistical issues at the beginning of the outbreak restricted enrollment of cases and controls, and the final sample size may have reduced the study power; thus, the ORs and 95\% Cls should be critically interpreted, even when the associations were not statistically significant. Non-differential misclassification of exposure may have occurred as it was based on participants recall. Serological tests for niacin were not performed because there is no laboratory capacity in Mozambique, and we were unable to collect samples and send them abroad. Thus, our case definition, which is based on dermatological features only, may have led to a selection bias because of potential inclusion of controls who had certain degree of niacin deficiency with no dermatological signs. If present, this bias would have reduced our capacity of detecting associations between the pattern of food consumption and pellagra but would not invalidate those associations that we found. Finally, we possibly underestimated pellagra in children $<5$ years because dermatological symptoms are usually not classic in children. ${ }^{4}$

However, the findings, which were supported by in-depth interviews, allowed us to conclude that people in Nhamatanda were already eating poorly even before the cyclone hit. Cyclone Idai however served as a trigger to reduce niacin consumption below the threshold that protected them from pellagra, by further limiting availability and access to sufficient quantity and quality of food. Improvement food availability, provision, and intake must be a priority to prevent pellagra from becoming endemic and to limit the emergence of other negative health outcomes. Further investigation is required to determine the prevalence of niacin and other micronutrient deficiency and their relationship with dietary intake in Nhamatanda and elsewhere in Mozambique where people often suffer from malnutrition.

Received October 9, 2020. Accepted for publication December 30, 2020.

Published online April 12, 2021.

Acknowledgments: The National Institute of Health of Mozambique, through its cooperation partners in the post-cyclone ldai emergency, funded this study. We gratefully appreciate the technical and logistical support received from the CIOB-Beira and Provincial Health Directorate of Sofala. We also appreciate the support from the Instituto Nacional de Saúde, Universidade Licungo, Conselho Nacional de Desenvolvimento Científico e Tecnológico (CNPq), and Instituto de Saúde Coletiva at the Universidade Federal da Bahia, Brazil. We would like to thank all the people who dedicated their time to the project and all the subjects in the study for their willingness to participate. Special thanks to Antônio Randinho and Manuel Brito (ClOB-Beira) who transported us tirelessly to the countryside.

Authors' addresses: Vánio André Mugabe, Instituto de Saúde Coletiva, Universidade Federal da Bahia, Salvador, Brazil and Universidade Licungo, Quelimane, Mozambique, E-mail: vandremu@gmail.com. Arlete Mahumane, Crescêncio Sequeira Nhabomba, Unicia Nyamula, Angélica Sotomane, and Benigno Canze, Centro de Investigação Operacional da Beira, Instituto Nacional de Saúde, Mozambique, E-mails: cynthiwe@ icloud.com, nhabombacrescencio@gmail.com, uniciacelestechibale@gmail. com, asotomane@gmail.com, and nigo755@gmail.com. Cynthia Semá Baltazar, Neusa Fataha, Osvaldo Frederico Inlamea, and Eduardo Samo Gudo, Departamento de Vigilância, Instituto Nacional de Saúde, Maputo, Mozambique, E-mails: cynthiasema@yahoo.com, neusafataha@gmail.com, osvaldosdc@gmail.com, and esamogudoj@gmail.com. Érika Valeska Rossetto, MassGenics, Assigned to Mozambique Centers for Disease Control Prevention, Maputo, Mozambique, E-mail: osc6@cdc.gov. Wilson Irugula, Direcção Provincial de Saúde de Sofala, Beira, Mozambique, E-mail: winrugula@gmail.com. Uriel Kitron, Emory University, Atlanta, GA, E-mail: ukitron@emory.edu. Guilherme Sousa Ribeiro, Instituto de Saúde Coletiva,
Universidade Federal da Bahia, Salvador, Brazil, Faculdade de Medicina, Universidade Federal da Bahia, Salvador, Brazil, and Fundação Oswaldo Cruz, Salvador, Brazil, E-mail: guilheme.ribeiro@fiocruz.br.

\section{REFERENCES}

1. Team TS, Schott T, Landsea C, Hafele G, Lorens J, Thurm H, Ward B, Willis M, Zaleski W, 2009. The Saffir-Simpson Hurricane Wind Scale. Available at: https://origin.www.nhc.noaa.gov/ pdf/sshws.pdf. Accessed August 21, 2020.

2. INE, 2017. Censo 2017. Available at: http://www.ine.gov.mz/ivcenso-2017/ic-censo-2017/view. Accessed April 10, 2020.

3. WHO, 2000. Pellagra and its Prevention and Control in Major Emergencies. Geneva, Switzerland: World Health Organization. Available at: http://www.who.int/nutrition/publications/emergencies/ WHO_NHD_00.10/en/. Accessed August 2020.

4. Naveen KN, Pai W, Bagalkot P, Kulkarni V, Rashme P, Athanikar SB, 2013. Pellagra in a child - a rare entity. Nutrition 29: 1426-1428.

5. Segula D, Banda P, Mulambia C, Kumwenda JJ, 2012. Case report a forgotten dermatological disease. Malawi Med J 24: 19-20.

6. Hersov LA, Rodnight R, 1960. Hartnup disease in psychiatric practice: clinical and biochemical features of three cases. $J$ Neurol Neurosurg Psychiatry 23: 40-45.

7. Hegyi J, Schwartz RA, Hegyi V, 2004. Pellagra: dermatitis, dementia, and diarrhea. Int J Dermatol 43: 1-5.

8. Berdanier CD, 2019. History of nutrition corn, niacin, and the history of pellagra. Nutr Today 54: 283-288.

9. Matapandeu G, Dunn SH, Pagels P, 2017. An outbreak of pellagra in the kasese catchment area, Dowa, Malawi. Am J Trop Med Hyg 96: 1244-1247.

10. Johnson ES, Marston JM, 2020. The experimental identification of nixtamalized maize through starch spherulites. J Archaeol Sci 113: 105056.

11. USDA, 2019. U.S. Household Food Security Survey Module: ThreeStage Design, With Screeners Economic Research Service, USDA September 2012. Available at: https://www.ers.usda.gov/topics/ food-nutrition-assistance/food-security-in-the-us/survey-tools. aspx\#adult. Accessed April 7, 2020.

12. ACC/SCN (UNITED NATIONS ADMINISTRATIVE COMMITTEE ON COORDINATION, NUTRITION) S-CO, 1996. Refugee Nutrition Information System (RNIS), No. 14 - Report on the Nutrition Situation of Refugees and Displaced Populations (UNSSCN, 1996, 45 p.). Available at: http://www.nzdl.org/ gsdlmod?e=d-00000-00—off-0fnl2.2-00-0—-0-10-0-0Odirect-10-4-0-11-11-ptZz-pt-50-20-preferences00-0-1-00-0-4-0-0-11-10-0utfZz-8-00\&cl=CL2.9.1\&d= HASH01a8c7da9e5e377c78dbc0a4\&gt=2. Accessed April 7, 2020.

13. Malfait P, Moren A, Dillon JC, Brodel A, Begkoyian G, Etchegorry MG, Malenga G, Hakewill P, 1993. An outbreak of pellagra related to changes in dietary niacin among mozambican refugees in Malawi. Int J Epidemiol 22: 504-511.

14. Seal AJ, Creeke PI, Dibari F, Cheung E, Kyroussis E, Semedo P, Van Den Briel T, 2007. Low and deficient niacin status and pellagra are endemic in postwar Angola. Am J Clin Nutr 85: 218-224.

15. Ginnaio $M, 2011$. Pellagra in late nineteenth century Italy: effects of a deficiency disease. Population 66: 583-609.

16. Mcintyre L, Glanville NT, Raine KD, Dayle JB, Anderson B, 2003. Do low-income lone mothers compromise their nutrition to feed their children? 168: 686-691.

17. Koch SL, 2019. Gender and Food: A Critical Look at the Food System. Available at: https://books.google.co.mz/books?id= mo2ADwAAQBAJ\&pg=PA64\&lpg=PA64\&dq=women+are+ more+likely+to+deprive+themselves+of+food+to+feed+their+ children\&source=bl\&ots=7G8ty1aTJX\&sig=ACfU3U2Rsq8CMs 4 luN9x7mMuBR8Ai9edeA\&hl=en\&sa=X\&ved=2ahUKEwiisp3Q1 tfoAhWyQ0EAHSXSAs8. Accessed August 2020.

18. Brenton BP, 2000. Pellagra, sex and gender: biocultural perspectives on differential diets and health. Nutr Anthropol 23: 20-24.

19. Shibata K, Toda S, Shibata K, Toda S, 2014. Effects of sex hormones on the metabolism of tryptophan to niacin and to serotonin in male rats. Biosci Biotech Biochem 61: 1200-1202. 